\title{
Miopia aguda induzida por topiramato: relato de dois casos
}

\section{Acute myopia induced by topiramate: 2 cases report}

Kathy Dadam Sgrott ${ }^{1}$, André Luis Momm da Silva', Luiz Felipe Hagemann², Vilmar Müller³, Fernando César Ludwig ${ }^{4}$

\section{ResUmo}

A miopia aguda pode ser desencadeada pelo uso de medicações sistêmicas, dentre elas, o anticonvulsivante topiramato. Este trabalho descreve dois casos de pacientes jovens com quadro agudo bilateral de miopia induzida por terapia com topiramato para controle de síndrome depressiva, fazendo relação com casos semelhantes descritos na literatura e revisão bibliográfica pertinente.

Descritores: Miopia/induzido quimicamente; Anticonvulsivantes/efeitos adversos; Relatos de casos

\section{Abstract}

The acute myopia can be caused by the use of systemic medications, such as topiramate. This study describes two cases of young patients with acute bilateral myopia induced by topiramate therapy to control depressive syndrome, making relation with similar cases described in literature and bibliographic review.

Keywords: Myopia/chemically induced; Anticonvulsants/adverse effects; Case report

\footnotetext{
${ }^{1}$ Médicos Residentes do Hospital de Olhos de Blumenau - HOB - Blumenau (SC), Brasil;

${ }^{2}$ Preceptor Chefe da Residência Médica do Hospital de Olhos de Blumenau - HOB - Blumenau (SC), Brasil;

${ }^{3}$ Chefe do Setor de Cirurgia Refrativa do Hospital de Olhos de Blumenau - HOB - Blumenau (SC), Brasil;

${ }^{4}$ Chefe do Setor de Lentes de Contato e Refratometria do Hospital de Olhos de Blumenau - HOB - Blumenau (SC), Brasil.

Os autores declaram inexistir conflitos de interesse.
}

Recebido para publicação em: 26/10/2009 - Aceito para publicação em 28/6/2011 


\section{INTRODUÇÃO}

$\mathbf{0}$ topiramato é um anticonvulsivante de amplo espectro, classificado como monossacarídeo sulfamato substituído. Recentemente, tem sido usado no manejo de migrânea, síndrome depressiva, dor neuropática e obesidade, em detrimento de seu uso para o controle da epilepsia, visto que é prescrito apenas em casos refratários ${ }^{(1)}$. Alguns relatos têm sido publicados relacionando-os a efeitos oftalmológicos adversos. As alterações oftalmológicas não são comuns, sendo mais alarmante o quadro de miopia aguda associada a glaucoma de ângulo fechado bilateral ${ }^{(2)}$.

\section{Relato Caso 1}

B.B.P., 30 anos, sexo feminino, branca, costureira, procurou o serviço de emergência do Hospital de Olhos de Blumenau com queixa de baixa acuidade visual (BAV) súbita para longe, bilateral, nas últimas 48 horas, mantendo visão normal para perto. A paciente negava antecedentes oftalmológicos e referia emetropia anteriormente. Os antecedentes médicos gerais incluíam síndrome depressiva em tratamento com Pondera ${ }^{\circledR}$ e, há sete dias, iniciado com topiramato na dose de $50 \mathrm{mg} /$ dia.

Ao exame inicial sua acuidade visual sem correção (AV) era em ambos olhos $(\mathrm{AO})<20 / 400$. O exame pupilar era normal bilateralmente, sem sinal de defeito pupilar aferente. À biomicroscopia apresentava câmara anterior ligeiramente rasa, tonometria dentro dos limites da normalidade: olho direito (OD) $16 \mathrm{mmHg}$ e olho esquerdo (OE) $14 \mathrm{mmHg}$. A refração dinâmica resultou em OD -7,00 -0,50 75 e, em OE -6,00 DE, AO com AV de 20/20. A refração estática após cicloplegia com ciclopentolato revelou em OD -6,75-0,50 75 e OE $-6,00-0,5030^{\circ}$ e a AV com a correção foi de 20/20 em ambos os olhos. A fundoscopia assim como a retinografia fluorescente apresentaram estrias retinianas, mais precisamente na membrana limitante interna, bilaterais.

A medicação foi descontinuada.

Em seguimento, após 10 dias, apresentou melhora do quadro. A refração estática, após ciclopentolato, revelou em OD +0,50 (20/25) e OE +0,50 (20/30), tonometria dentro dos limites da normalidade $\mathrm{AO}$ e sem particularidades à biomicroscopia.

\section{Relato Caso 2}

S.V.D., 36 anos, sexo feminino, branca, professora, procurou o serviço de emergência do Hospital de Olhos de Blumenau com queixa de BAV súbita para longe, bi- lateral, nas últimas 24 horas, mantendo visão para perto. Sem antecedentes oftalmológicos, referia emetropia prévia. Os antecedentes médicos gerais incluíam síndrome depressiva em tratamento com fluoxetina, rivotril ${ }^{\circledR}, \mathrm{e}$, há quatro dias, havia iniciado uso de topiramato na dose de $50 \mathrm{mg} /$ dia.

Em exame inicial sua acuidade visual era, sem correção, em OD 20/70 e em OE 20/100. O exame pupilar era normal bilateralmente, sem sinal de defeito pupilar aferente. À biomicroscopia não apresentava alterações, tonometria dentro dos limites da normalidade: $12 \mathrm{mmHg}$ em ambos os olhos. A refração dinâmica resultou em OD -1,00 -0,50 60 e em OE -1,00 -0,75 105, AO com AV de 20/30. A refração estática após cicloplegia com ciclopentolato revelou em OD -1,00 -0,75 60 e OE $-1,25-0,75105$ e a AV com a correção foi de 20/30 em ambos os olhos. A fundoscopia não evidenciou alterações. A retinografia fluorescente evidenciou área de gliose em região temporal de OE, sem relação clínica com o quadro da paciente.

A medicação foi descontinuada.

Em seguimento, após 10 dias, apresentou melhora do quadro, a refração estática após ciclopentolato revelou em OD +0,50 (20/25) e OE +0,75 (20/25), tonometria dentro dos limites da normalidade AO: $12 \mathrm{mmHg}$ em OD e $11 \mathrm{mmHg}$ em OE; sem particularidades à biomicroscopia.

\section{Discussão}

Diversos relatos têm sido publicados relacionando o topiramato a efeitos secundários patológicos ${ }^{(3)}$, tanto em adultos quanto em crianças, evidenciados no primeiro mês de tratamento ${ }^{(4,5)}$. Entre os efeitos adversos sistêmicos estão: anorexia, perturbações do humor, nefrolitíase, acidose metabólica e redução da função hepática. Os achados oftalmológicos, apesar de incomuns, englobam uma síndrome composta por glaucoma secundário de ângulo fechado e miopia agu$\mathrm{da}^{(6)}$.

O mecanismo de ação desta síndrome não é totalmente conhecido. Alguns autores acreditam que o processo acontece por efusão uveal devido à edema do corpo ciliar, com consequente anteriorização do diafragma irido-cristaliniano, levando à diminuição da profundidade da câmara anterior e fechamento do seio camerular, sem bloqueio pupilar ${ }^{(7)}$.

A efusão uveal induzida por drogas é extremamente rara, embora tenha sido relatada também com o uso de derivados das sulfas, como a acetazolamida, 


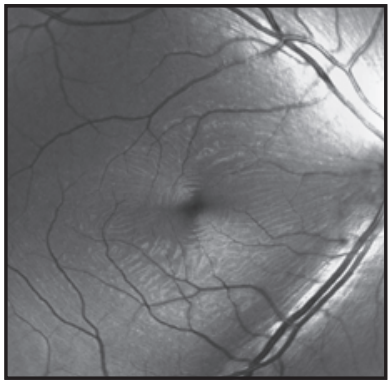

Figura 1: Retinografia fluorescente red free OD

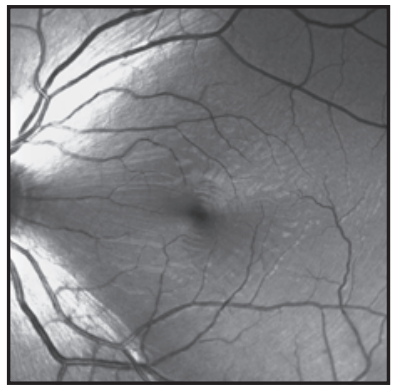

Figura 2: Retinografia fluorescente red free $\mathrm{OE}$ hidroclortiazida, sulfametoxazol e os inibidores seletivos da recaptação de serotonina.

Outros autores sugerem que a miopia aguda pode também estar relacionada com a fraca inibição da anidrase carbônica causada pelo topiramato, com o edema do próprio cristalino e o espasmo acomodativo ${ }^{(8-}$ ${ }^{10)}$. O espasmo acomodativo considerado na etiologia da síndrome tem fraca credibilidade, pois, assim como nos casos descritos, os relatos mostraram que após a cicloplegia os pacientes permaneciam míopes

Hook et al. ${ }^{(11)}$ demonstraram através de estudos ecobiométricos com A-scan que pacientes que desenvolveram miopia com o uso de sulfonamidas, tiveram sua miopia relacionada a espessamento cristaliniano em $87 \%$ dos casos e a deslocamento anterior do cristalino em $13 \%$, o que sugere uma combinação de mecanismos. Como o mecanismo de fechamento angular não envolve bloqueio pupilar, iridectomias periféricas e o uso de mióticos tópicos não são efetivos para tratar o glaucoma secundário ao fechamento do ângulo. Se diagnosticada precocemente, a síndrome é reversível com a descontinuação da droga, assim como a administração de colírios antiglaucomatosos e hidratação vigorosa por curto período ${ }^{(12,13)}$. A normalização do quadro após suspensão da droga é observada em torno de três a sete dias - a meia-vida da droga é de 20 a 30 horas e sua excreção é principalmente renal ${ }^{(14,15)}$.

\section{REFERÊNCIAS}

1. Bhattacharyya KB, Basu S. Acute myopia induced by topiramate: report of a case and review of the literature. Neurol India. 2005;53(1):108-9. Comment in Neurol India. 2005;53(2):243; author reply 243.

2. Stangler F, Prietsch RF, Fortes Filho JB. Glaucoma agudo bilateral em paciente jovem secundário ao uso de topiramato: relato de caso. Arq Bras Oftalmol. 2007;70(1):133-6.

3. Rhee DJ, Goldberg MJ, Parrish RK. Bilateral angle-closure glaucoma and ciliary body swelling from topiramate. Arch Ophthalmol. 2001;119(11):1721-3. Comment in Arch Ophthalmol. 2002;120(12):1775.

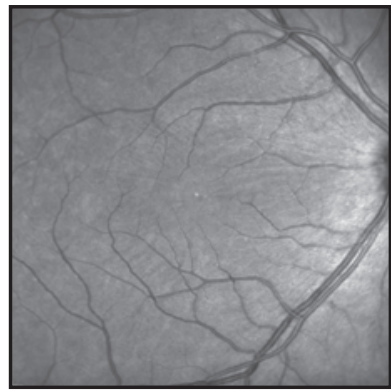

Figura 3: Retinografia fluorescente Infrared OD

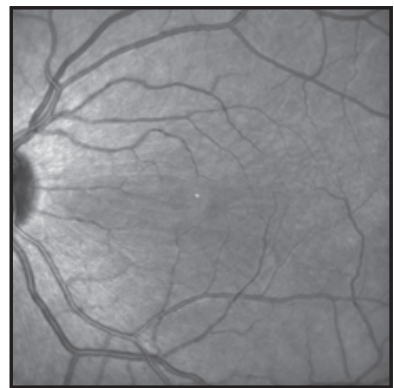

Figura 4: Retinografia fluorescente Infrared OE
4. Lachkar Y, Bouassida W. Drug-induced acute angle closure glaucoma. Curr Opin Ophthalmol. 2007;18(2):129-33.

5. Levy J, Yagev R, Petrova A, Lifshitz T. Topiramate-induced bilateral angle-closure glaucoma. Can J Ophthalmol. 2006;41(2):221-5.

6. Santaella RM, Fraunfelder FW. Ocular adverse effects associated with systemic medications: recognition and management. Drugs. 2007;67(1):75-93.

7. Craig JE, Ong TJ, Louis DL, Wells JM. Mechanism of topiramate-induced acute-onset myopia and angle closure glaucoma. Am J Ophthalmol. 2004;137(1):193-5

8. Desai CM, Ramchandani SJ, Bhopale SG, Ramchandani SS. Acute myopia and angle closure caused by topiramate, a drug used for prophylaxis of migraine. Indian J Ophthalmol. 2006;54(3):195-7.

9. Sachi D, Vijaya L. Topiramate induced secondary angle closure glaucoma. J Postgrad Med. 2006;52(1):72-3.

10. Chen TC, Chao CW, Sorkin JA. Topiramate induced myopic shift and angle closure glaucoma. Br J Ophthalmol. 2003;87(5):648-9.

11. Hook SR, Holladay JT, Prager TC, Goosey JD. Transient myopia induced by sulfonamides. Am J Ophthalmol. 1986;101(4):495-6.

12. Boentert M, Aretz H, Ludemann P. Acute myopia and angleclosure glaucoma induced by topiramate. Neurology. 2003;61(9):1306. Comment in Neurology. 2004;63(4):762; author reply 762 .

13. Thambi L, Kapcala LP, Chambers W, Nourjah P, Beitz J, Chen $\mathrm{M}, \mathrm{Lu}$ S. Topiramate-associated secondary angle-closure glaucoma: a case series. Arch Ophthalmol. 2002;120(8):1108. Comment on Arch Ophthalmol. 200;119(8):1210-1.

14. Fraunfelder FW, Fraunfelder FT, Keates EU. Topiramateassociated acute, bilateral, secondary angle-closure glaucoma. Ophthalmology. 2004;111(1):109-11.

15. Almeida HG, Cohen R, editores. Glaucomas secundários. 2a ed. São Paulo: Roca; 2006. 